\title{
An Unusual Presentation of Leptospirosis in an urban setting as an overlooked cause of multiorgan failure
}

\author{
Md. Tarek Alam ${ }^{1 *}$, Sadia Saber ${ }^{2}$, Rafa Faaria Alam³, Mohammad Monower Hossain ${ }^{4}$

\begin{abstract}
:
Leptospirosis is a life-threatening zoonotic disease of global distribution. It has variable presentation ranging from mild febrile illness to life-threatening complications like acute renal failure, acute hepatic failure, pulmonary hemorrhages and cardiac arrhythmias. We present a case of flu-like illness contracted by working at a poorly hygienic place where the patient experienced progressive pulmonary-renal syndrome two days after admission. Index suspicion
\end{abstract} \\ and early treatment remains the mainstay of this life threatening zoonotic disease.
}

Key Words: Leptospirosis, Weil's disease, Acute pulmonary hemorrhage, Pulmonary-renal syndrome, Microscopic agglutination test (MAT).

\section{Background:}

Leptospirosis is a potentially fatal zoonotic disease which is caused by the pathogenic spirochete of genus leptospira ${ }^{1}$. It is characterized by a broad spectrum of clinical manifestations which include subclinical infection, self-limited anicteric febrile illness and a severe and potentially fatal illness known as Weil's syndrome that presents with jaundice, renal failure and haemorrhage ${ }^{2}$. It is a disease of worldwide distribution. Human infection occurs either through the direct contact with infected animals or more of the times through the contact with water or soil contaminated with urine of infected rodents or animals ${ }^{1}$. The definitive diagnosis of leptospirosis is based either on isolation of the organism from clinical specimens or a fourfold or greater rise in antibody titre in the convalescence phase sera. The management of leptospirosis includes supportive measures and the use of penicillin or tetracycline to eradicate the organism. The efficacy of antibiotics remains controversial although some studies showed that administration of intravenous penicillin or doxycycline at an early stage of the illness was beneficial².

\section{CASE REPORT:}

A previously healthy 55-year-old man came to our emergency department due to fever, generalized myalgia, lethargy and right hypochondrial pain for one-week. He was anuric for 2 days. On examination he was found to be febrile $\left(38^{\circ} \mathrm{c}\right)$,

1. Prof of Medicine, Bangladesh Medical College \& Hospital, Dhanmondi, Dhaka.

2. Asstt. Prof of Medicine, Bangladesh Medical College \& Hospital, Dhanmondi, Dhaka

3. Honorary Medical officer, Bangladesh Medical College \& Hospital, Dhanmondi, Dhaka

4. Medical Officer of Medicine, Bangladesh Medical College \& Hospital, Dhanmondi, Dhaka.

\section{*Corresponding Author:}

Dr. Md. Tarek Alam, MBBS, MD (USA)

Prof of Medicine,

Bangladesh Medical College \& Hospital, Dhanmondi, Dhaka.

Email: mtarekalam@hotmail.com dehydrated, jaundiced, tachycardic (pulse rate of 102/min) and a blood pressure of $110 / 70 \mathrm{mmHg}$. He had generalized muscle tenderness. The skin turgor was senile without rashes or wounds over chest, abdomen or back. His conjunctivae were normal and his sclera was icteric. Abdominal examination revealed right hypochondriaI tenderness with hepatomegaly but no other organomegaly. His heart sound and breathing sounds were normal. The remainder of his physical examinations were unremarkable.

Laboratory results at his admission are given below:

\begin{tabular}{lc}
\hline Parameters & On Admission \\
\hline Haemoglobin $(\mathrm{g} / \mathrm{dl})$ & 7.60 \\
Total white cells count $\left(10^{9} / \mathrm{l}\right)$ & 8.00 \\
Platelets $\left(10^{9} / \mathrm{l}\right)$ & 80.00 \\
Sodium $(\mathrm{mmol} / \mathrm{l})$ & 135 \\
Potassium $(\mathrm{mmol} / \mathrm{l})$ & 3.63 \\
Creatinine $(\mathrm{mg} / \mathrm{dl})$ & 7.1 \\
Urea $(\mathrm{mg} / \mathrm{dl})$ & 240 \\
Total protein $(\mathrm{g} / \mathrm{l})$ & 51 \\
Total bilirubin $(\mathrm{mg} / \mathrm{dl})$ & 28.26 \\
Alkaline phosphatase $(\mathrm{IU} / \mathrm{l})$ & 84 \\
Alanine transaminase $(\mathrm{IU} / \mathrm{l})$ & 78 \\
\hline
\end{tabular}

Blood and urine cultures, blood film for malarial parasites, dengue serology, and Widal-weil Felix serology tests were all negative. Ultrasonography of the abdomen showed Hepatomegaly with hypoechoic parenchyma-suggesting hepatitis, bilateral swollen kidney - acute nephropathy, mildly enlarged prostate gland (volume $27 \mathrm{cc}$ ). The chest radiography showed borderline cardiomegaly, with no active lung lesion in both lung fields.

On hospital day 4 at midnight, the patient experienced progressive respiratory distress associated with confused consciousness and hemoptysis. The chest radiography showed generalized interstitial infiltrations in both lungs. Acute 
pulmonary hemorrhage with respiratory failure was considered. The patient was transferred to the intensive care unit where he received endotracheal intubation with mechanical ventilation.

According to the contact history of rats' infestations and clinical presentation of the patient including pharyngeal erythema, abdominal cramping pain, acute pulmonary hemorrhage, leptospirosis was suspected. Leptospirosis was confirmed by antibody test where $\operatorname{IgM}$ antibody came positive.

He was treated with aggressive fluid therapy and intravenous ceftriaxone $1 \mathrm{gm} \mathrm{I} / \mathrm{V} 12$ hourly. His symptoms and hepatic function improved 10 days later and his renal failure recovered without a need for dialysis. However his hemoglobin level and platelet counts remained low.

The patient stayed in the hospital for about one month due to multi-organ damage (pulmonary hemorrhage, acute kidney injury, jaundice and respiratory failure) and nosocomial infection (extended spectrum $\beta$-lactamases producing Klebsiella pneumoniae bacteremia, Pseudomonas aeruginosa pneumonia). After intensive treatment including blood transfusion and antibiotic therapy, his heart, lung, and renal functions improved. He was liberated from mechanical ventilation and recovered uneventfully. There were no permanent squeals at a four month follow-up.

\section{DISCUSSION:}

Leptospirosis is a zoonosis of worldwide distribution caused by infection with $L$. interrogans, a pathogenic spirochete. The organism infects a variety of animals, especially rodents and animals associated with farming. Humans represent only incidental infection usually via work-related contact through skin or mucous membranes, typically after exposure to water or soil contaminated with urine from an infected animal or via drinking of or bathing in contaminated water. The main occupational groups at risk are farm workers, field agricultural workers, plumbers, sewer workers, sanitation workers and military troops.

Leptospira are spiral-shaped, thin, motile organisms with flagella. The most common servers are ictero haemorrhagiae, which are usually found in rats (Rattus norvegicus). Urinary shedding of organisms from infected animals is the most significant source of Leptospira spp. because the spirochetes can persist for long periods of time in the renal tubules.

The patient became infected at a poorly hygienic place with rodent infestation. He presented diverse symptoms from fever, myalgia, and epigastric tenderness to multi-organ dysfunction including pulmonary hemorrhage, acute kidney injury, jaundice and respiratory failure.

Pulmonary involvement in leptospirosis ranges from 20-70\% of patients ${ }^{3}$. Pulmonary symptoms occur in both anicteric and icteric forms of leptospirosis. Pulmonary hemorrhage is the most serious pulmonary manifestation. Respiratory symptoms usually appear between the fourth and sixth day of disease and may lead to death in less than 72 hours. Mortality rates can be as high as $30-60 \%{ }^{3}$.
Concerning the pathogenesis of lung injury in leptospirosis, two main mechanisms are suggested-septicemic and immune. Humans typically become ill seven to 12 days after exposure to leptospira. The first stage is called the septicemic phase (leptospiremic phase) because the bacteria may be isolated from blood cultures and cerebrospinal fluid (CSF). This phase is characterized by a nonspecific flulike illness with sudden onset of high fever, headache, myalgia - classically involving the Para spinal, calf and abdominal muscles ${ }^{4}$ and conjunctival suffusion. Conjunctival suffusion (reddening of the eye surface) is a characteristic physical finding in leptospirosis, and its presence in a patient with a nonspecific febrile illness should raise suspicion for diagnosis.

The second stage is called the immune phase (leptospiruric phase) when circulating antibodies can be detected and the bacteria can be isolated from the urine. This stage occurs as a result of the body's immunologic response by producing immunoglobulin $\mathrm{M}$ antibodies and can last longer than one month. During this stage, specific organ damage can be observed. Aseptic meningitis is one of the most important clinical syndromes that can occur in $80 \%$ of patients during the immune phase. Renal symptoms, such as uremia, azotemia, pyuria and hematuria, may occur. Pulmonary manifestations, although usually benign, can be potentially life threatening and range from chest pain, cough and dyspnea to pulmonary hemorrhage or acute respiratory distress syndrome. An increase in liver enzymes (up to five times normal) with a disproportionately high total bilirubin has been described as a prognostic indicator in leptospirosis ${ }^{5}$. Varying degrees of jaundice, pancreatitis, hepatomegaly and myocarditis can also occur.

Weil's disease is the most severe form of leptospirosis. Patients can present with high fever $\left(>40^{\circ} \mathrm{C}\right)$, significant jaundice, renal failure, hepatic necrosis, pulmonary involvement, cardiovascular collapse, neurologic changes and hemorrhagic diathesis, with a variable clinical course. Weil's disease can occur at the end of the first stage and peaks during the second stage but can occur at any time during acute leptospirosis as a single, progressive illness.

Acute renal failure is one of the most common complications of severe leptospirosis. Renal leptospirosis is usually described as a combination of acute tubular damage and interstitial nephritis. Leptospira outer membrane proteins (OMPs) may elicit tubular injury and inflammation ${ }^{6}$. Renal function returns typically to normal after early treatment. A case-control study of Leptospirosis in Taiwan showed prominent presentations with increased incidence of bilateral enlarged kidneys, sterile pyuria, and hypokalemia as compared with excluded cases ${ }^{7}$.

Renal disease associated with pulmonary hemorrhage is seen in a variety of clinical disorders ${ }^{8}$. Common pulmonary-renal syndromes including anti-GBM disease and ANCA associated vasculitis. The infection is the differential diagnosis as well. Anti-GBM disease, anti-ANCA-related vasculitis and leptospirosis have the similar constitutional symptoms such as fever, arthralgia, myalgia and flu-like syndrome. Nally and associates demonstrated the deposition 
of immunoglobulin $\mathrm{G}$ ( $\mathrm{IgG})$, immunoglobulin $\mathrm{A}(\operatorname{IgA})$ and Complement 3(C3) along the alveolar basement membrane of infected guinea pigs in a leptospirosis animal model, which may be similar to the pattern that seen in Good pasture's syndrome ${ }^{9}$.

Hepatic dysfunction is usually mild and reversible. Liver dysfunction in severe leptospirosis can be seen as conjugated serum bilirubin levels may increase to above $80 \mathrm{mg} / \mathrm{dL}$, accompanied by modest elevations in transaminases, which rarely exceed $200 \mathrm{U} / \mathrm{L}^{10}$.

Variable degrees of thrombocytopenia have been reported with leptospirosis. The pathogenesis of thrombocytopenia and hemorrhagic diathesis in leptospirosis is not well understood.

Overall, Weil's syndrome has a mortality rate of $5 \%$ to $10 \%$. Important causes of death include renal failure, cardiopulmonary failure and widespread hemorrhage ${ }^{11}$.

A detailed exposure history taken from the suspected patients is critical for diagnosis. Biochemical, hematologic and urinalysis parameters in leptospirosis are variable and non-specific. Classic Weil's disease may demonstrate elevated blood urea nitrogen, serum creatinine, bilirubin, SGOT, and SGPT. In hematologic abnormalities, thrombocytopenia appears independently of disseminated intravascular coagulation and may be concurrent with severe endotoxin injury of leptospirosis ${ }^{3}$. Thrombocytopenia develops in up-to $50 \%$ of patients and usually correlates with nonoligouric renal failure as a possible early hint of leptospirosis ${ }^{12}$.

The Microscopic agglutination test (MAT) is the gold standard for serologic diagnosis of leptospirosis. The criterion for a positive MAT is a four-fold increase in antibody titer or a conversion from seronegative to a titer of $\geq 1: 100$. Owing to the high inter-laboratory variation in MAT, the commercially available assays that can detect IgM antibodies during the first week of illness are on the rise ${ }^{13}$.

The vast majority of infections with leptospira are self-limiting, and it remains controversial if antimicrobials produce benefit in cases of mild leptospirosis without end-organ damage. Current antimicrobial treatments for leptospirosis include doxycycline, penicillin, and the third-generation cephalosporin. All regimens were observed to be therapeutically equivalent in one randomized trial ${ }^{14}$. For moderate/severe leptospirosis, penicillin (1.5 million units IV Q6h) or ceftriaxone (1g IV bid) or cefotaxime (1g IV Q6h) should be used. Before the diagnosis can be confirmed, broad-spectrum parenteral antibiotic is required for suspicion manifesting as multiorgan system dysfunction ${ }^{1}$. Mild disease should be treated with oral antibiotics, in particular doxycycline (100mg po bid $)^{1}$. It is also reported as an effective prophylaxis for soldiers training in endemic areas ${ }^{15}$.

The use of steroids in patients with leptospirosis has not been well established. In the current case, the improvement of the patient's renal dysfunction, thrombocytopenia and hemoptysis may be attributed to the introduction of steroids. Several case reports have described the beneficial effects of glucocorticoids in severe leptospirosis with pulmonary hemorrhage $^{16}$, thrombocytopenia ${ }^{17}$ and renal failure ${ }^{17,18}$.

Public health measures to prevent and reduce leptospirosis include identification of contaminated water sources, rodent control, and prohibition of swimming in waters where risk of infection is high and informing persons of the risk involved in recreational water activities.

\section{CONCLUSION:}

In conclusion, leptospirosis has recently come to international attention as a globally important reemerging infectious disease in not only developing countries but in industrialized nations as well. It is a challenge to diagnose the infection of Leptospira because of the diverse presentations. The most common triad is fever, jaundice, and acute renal failure. In addition to hepatic and renal dysfunction, leptospirosis should be seriously considered in patients with pulmonary symptoms. Early awareness of the distinct presentations of leptospirosis and prompt antibiotic therapy can dramatically save the patients.

\section{References:}

1. Paul, N.V., et al. (2001) Leptospira Species (Leptospirosis). Infectious Diseases and Their Etiologic Agents.

2. Farr RW. Leptospirosis. Clin Infect Dis 1995;21(1):1-6

3. Dolhnikoff M, Mauad T, Bethlem EP, Carvalho CR. Leptospiral pneumonias. Curr Opin Pulm Med 2007;13: 230-5.

4. Levett PN, Haake DA: Leptospira species (leptospirosis). In: Principles and Practice of Infectious Diseases. Edited by: Mandell GL, Bennett JE, Dolin R. 2010, Philadelphia: Churchill Livingstone Elsevier, 3059-3065.

5. Chang ML, Yang CW, Chen JC, Ho YP, Pan MJ, Lin $\mathrm{CH}$ et al.Disproportional exaggerated aspartate transaminase is a useful prognostic parameter in late leptospirosis. World J Gastroenterol. 2005, 11 (35): 5553-5556.

6. Yang, CW. Leptospirosis renal disease: understanding the initiation by Toll-like receptors. Kidney International 2007; 72: 918-25.

7. Rodriguez W, Hanania N, Guy E, Guntupalli J. Pulmonary renal syndromes in the intensive care unit. Crit Care Clin 2002; 18(4): $881-95$.

8. Kishimoto M, Brown JD, Chung HH, Howman S. Leptospirosis misdiagnosed as pulmonary-renal syndrome. Am J Med Sci 2004; 328(2):116-20.

9. Nally JE, Chantranuwat C, Wu XY. Alveolar septal deposition of immunoglobulin and complement parallels pulmonary hemorrhage in a guinea pig model of severe pulmonary leptospirosis. Am J Pathol 2004; 164:1115-27.

10. Edwards GA, Domm BM: Leptospirosis. Med Times. 1966, 94 (9): 1086-1095.

11. Human Leptospirosis: Guidance for Diagnosis, Surveillance, and Control. 2003, Geneva: World Health Organization

12. Yang HL, Jiang XC, Zhang XY.Thrombocytopenia in the experimental leptospirosis of guinea pig is not related to disseminated intravascular coagulation. BMC Infect Dis 2006; 6:19.

13. Bajani MD, Ashford DA, Bragg SL. Evaluation of four commercially available rapid serologic tests for diagnosis of leptospirosis. J Clin Microbiol 2003; 41:803-9. 
14. Suputtamongkol Y, Niwattayakul K, Suttinont C. An open, randomized, controlled trial of penicillin, doxycycline, and cefotaxime for patients with severe leptospirosis. Clin Infect Dis 2004; 39(10):1417-24.

15. Brett-Major DM, Coldren R. Antibiotics for leptospirosis. Cochrane Database Syst Rev 2012; 15: 2:CD008264.

16. Shenoy VV, Nagar VS, Chowdhury AA, Bhalgat PS, Juvale NI: Pulmonary leptospirosis: an excellent response to bolus methylprednisolone. Postgrad Med J. 2006, 82 (971): 602-606.
17. Wagenaar JF, Goris MG, Partiningrum DL, Isbandrio B, Hartskeerl RA, Brandjes DP et al.Coagulation disorders in patients with severe leptospirosis are associated with severe bleeding and mortality. Trop Med Int Health. 2010, 15 (2): 152-159.

18. Dursun B, Bostan F, Artac M, Varan HI, Suleymanlar G.Severe pulmonary haemorrhage accompanying hepatorenal failure in fulminant leptospirosis. Int J Clin Pract. 2007, 61 (1): 164-167. 